\title{
Flexible Microstructuring of Thin Films Using Multi-beam Interference Ablation with Ultrashort Lasers
}

\author{
Bogdan VOISIAT $^{* 1}$, Mindaugas GEDVILAS ${ }^{* 1}$, Simonas INDRIŠIŪNAS ${ }^{* 1}$ and Gediminas RAČIUKAITIS ${ }^{* 1}$ \\ ${ }^{* 1}$ Center for Physical Sciences and Technology, Savanoriu Ave. 231, LT-02300, Vilnius, Lithuania \\ E-mail: mgedvilas@ar.fi.lt
}

\begin{abstract}
Interference of laser beams with the high pulse energy opens an opportunity to direct structuring over large areas. We report results of the laser beam interference ablation (LBIA) of thin metal films on the glass substrate irradiated with picosecond and nanosecond lasers. The laser beam was split into four and six beams by using the diffractive optical element. The 4F imaging system was used to produce interference pattern on the surface of the metal film. Regular structures with a period of $5 \mu \mathrm{m}$ were produced in thin films of various metals which can be used for filtering infrared or terahertz radiation. The structure was extended by translation of the workpiece between laser pulses. Interference of multiple laser beams is capable of forming a variety of interference patterns, while selection of the laser wavelength as well as imaging optics open possibilities to control the period of the structures down to the sub-micrometer range.

DOI:10.2961/jlmn.2011.03.0002
\end{abstract}

Keywords: thin films, multi-beam, interference ablation, diffractive optical element

\section{Introduction}

Properties of the surface highly depend on its relief. The best example is a lotus leaf with the "self-cleaning", hydrophobic feature. Micro- and nano-structuring with specific patterns and at localized places is useful to control wettability, lubrication and other properties of the surface. Artificial structures borrowed from the nature are applied in automotive industry as well as in photonics and biomedical research. A new growing application area is frequency-selective surfaces [1, 2, 3], which are working as filters or antennas for electro-magnetic waves in $\mathrm{THz}$, infrared or visible range depending on the period and the feature size of the fabricated surface. They can be produced by laser structuring of metal films.

Laser direct writing (LDW) is a flexible method but the performance on practically large areas is too low when fine structures should be produced [4]. Laser-induced periodical surface structures, ripples, [5] are useful to control surface absorptivity [6], however, it is difficult to create a desirable shape.

Laser patterning using interference of several beams is capable of producing the sub-wavelength features not limited by a beam spot size and is an effective method of forming two-dimensional (2D) and three-dimensional (3D) structures [7]. The periodical structure can be controlled by changing an incidence angle of the beams, radiation wavelength the phase difference between the beams, polarization and intensity. 1D and 2D structures were achieved by combining several laser beams [8]. Holographic lithography by combining a few beams of a femtosecond laser and the controllable phase shift between them was used for formation of periodic structures in photo-polymers [9].

Different techniques are applied to produce the interfering field of laser radiation. In the Lloyd's schema, a part of the expanded beam is reflected from a tilted mirror and interferes with the incident light on a sample surface. The period of the patterns can be adjusted by an angle between the mirror and samples [10]. The schema is simple, but it can be used with the continuous wave or long pulse lasers. Compensation of temporal delay between separate beams is required to get interference with short laser pulses when the laser beam is split using partially reflecting beam-splitters $[11,12,13]$.

For ultra-short laser pulses $(<10 \mathrm{ps})$ a diffractive optical element (DOE) is preferable instead of mirrors, as DOE inclines the wave front in such a way that it remains perpendicular to the common optical axis and an overlap of the beams is ensured over the whole beam diameter. The optical path for all beams is exactly the same and the period of the structure is independent of the wavelength, as it is defined by the DOE period [14]. DOE has also recently become popular for splitting the laser beam into the desired (more than two) number of beams. Additional optics is applied to combine those beams to produce interference patterns [6].

The interfering laser beam with a high pulse energy was applied to direct laser ablation in bulk metals [15] and metal films [16, 17, 18] and $\mathrm{ZnO}$ films [19]. The optical absorbance of the metallic surface was enhanced by using interferometric laser surface patterning [20].

In this work, the high-pulse-energy picosecond laser was applied to produce interference patterns in thin metallic films by direct ablation. The laser beam interference ablation (LBIA) was tested as a method of laser patterning over large areas with a controllable shape.

\section{Experimental}

Two similar experimental setups with classical $4 \mathrm{~F}$ imagining systems described in [21, 22] for four- and sixbeam LBIA were used in the experiments and are shown in Fig. 1 and Fig. 2. The laser beam was split into 4 and 6 
beams by using DOEs. The parameters of the DOEs used in the experiments are given in Table 1.

Table 1 Parameters of DOE used in LBIA experiments.

\begin{tabular}{cccc}
\hline \# Beams & $\begin{array}{c}\text { Wavelength, } \\
\mathrm{nm}\end{array}$ & $\Lambda_{\mathrm{DOE}}, \mu \mathrm{m}$ & $\begin{array}{c}\text { Deflection } \\
\text { angle, deg }\end{array}$ \\
\hline 4 & 1064 & 30 & 2.9 \\
6 & 532 & 7 & 5 \\
\hline
\end{tabular}

The image of the DOE was translated to the workpiece surface by two lenses arranged in 4F imaging system. The first lens with focal length of $F_{1}$ was placed at this distance from DOE, and all beams propagated in parallel. The second lens was added at a distance equal to the sum of both focal lengths $F_{1}+F_{2}$. All beams were overlapped beneath the second lens, creating the interference patterns in the intersection area. The modulation period in the workpiece plane for 4-beam interference can be written as:

$$
\Lambda_{4}=M \frac{\Lambda_{\mathrm{DOE}}}{2},
$$

where $\Lambda_{\mathrm{DOE}}$ is a period of the DOE, $M=F_{2} / F_{1}$ is the magnification factor of $4 \mathrm{~F}$ imaging system. For the modulation period of 6-beam interference another equation should be used:

$$
\Lambda_{6}=M \Lambda_{\mathrm{DOE}} .
$$

The diaphragm was set between lenses to block the zero and higher orders of the diffracted beam, as it was found out that even low intensity in the zero-order beam significantly affected the period of interference modulation [23].

The piezoelectric 3-axis positioning system PIHera (Physical Instruments) was able to move $500 \mu \mathrm{m}$ in $x$ and $y$ direction and $250 \mu \mathrm{m}$ in $z$ direction with the minimal programmable step of $10 \mathrm{~nm}$. It was used for precise focusing of the beam and scanning the sample.

\subsection{Four-beam LBIA experimental setup}

Experimental setup for four-beam LBIA is shown in Fig.1. The picosecond laser Foxtrot (Ekspla Ltd.) with high pulse energy $(0.7 \mathrm{~mJ})$ was used in the experiments. The pulse duration at FWHM was 60 ps. The laser generated the $1064 \mathrm{~nm}$ radiation at the repetition rate of $4 \mathrm{kHz}$.

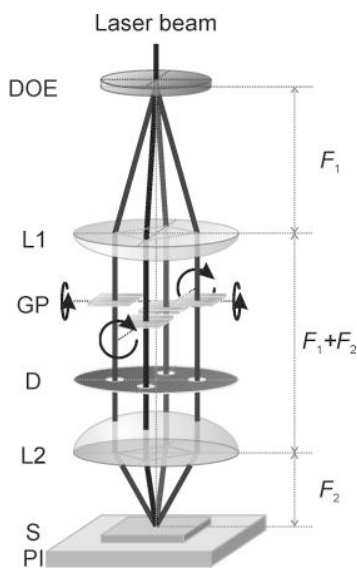

Fig. 1 Setup for LBIA experiments with four beams and 4F imaging system. DOE - diffractive optical element; L1 and L2 - spherical lenses with focal length of $F_{1}=75 \mathrm{~mm}$ and $F_{2}=25 \mathrm{~mm}$ respectively, $\mathrm{GP}$ - rotating glass plates to control phase and intensity of each beam, D - diaphragm, $\mathrm{S}$ - sample and PI - piezoelectric $x y z$ stage.
The laser was selected because of high pulse energy, and the laser fluence above the ablation threshold was maintained over a large focal spot. Single pulses or sequence of pulses were extracted from the pulse train employing a Pockels cell based pulse picker. The Pockels cell was also used as an attenuator to control the laser power. The spot irradiated by the laser beam with interference modulation was $475 \mu \mathrm{m}$ in diameter.

\subsection{Six-beam LBIA experimental setup}

Experimental setup for six-beam LBIA is shown in Fig. 2. The nanosecond laser NL220 (Ekspla Ltd.) with high pulse energy $(5.0 \mathrm{~mJ})$ was used in the experiments. The pulse duration at FWHM was 9 ns. The laser generated the $532 \mathrm{~nm}$ radiation at the repetition rate of $500 \mathrm{~Hz}$.

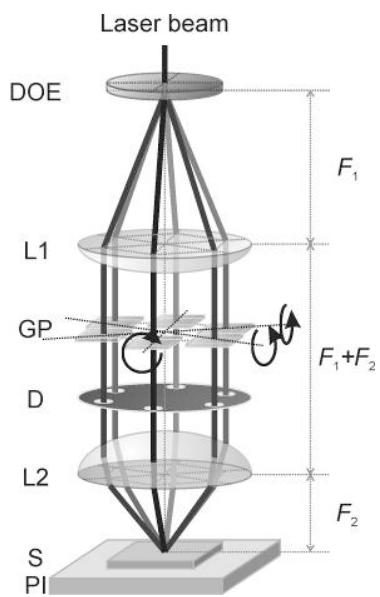

Fig. 2 Setup for LBIA experiments with six beams and 4F imaging system. DOE - diffractive optical element; L1 and L2 - spherical lenses with focal length of $F_{1}=75 \mathrm{~mm}$ and $F_{2}=50 \mathrm{~mm}$, respectively, $\mathrm{GP}-$ rotating glass plates to control phase and intensity of each beam, D - diaphragm, $\mathrm{S}$ - sample and PI - piezoelectric $x y z$ stage.

Glass plates were inserted in the optical path of the beams and could be tilted to adjust the phase difference between the laser beams and to control intensity of each beam. With the tilting accuracy of $0.1 \mathrm{deg}$ and thickness of the glass plate of $1 \mathrm{~mm}$ the accuracy of phase shifting was less than $30 \mathrm{~nm}$.

Periodical structures were formed in metal films deposited on a glass substrate. Six different metals were used in experiments: chromium, aluminum, silver, copper, gold, and nickel. Thickness of the metal films and substrates are presented in Table 2.

Table 2 Thickness of metal films and substrates.

\begin{tabular}{cccc}
\hline Metal film & $\begin{array}{c}\text { Film thickness, } \\
\mathrm{nm}\end{array}$ & Substrate & $\begin{array}{c}\text { Substrate thick- } \\
\text { ness, mm }\end{array}$ \\
\hline Chromium & 100 & Glass & 5 \\
Aluminum & 100 & Glass & 2.3 \\
Silver & 100 & Glass & 2.3 \\
Copper & 500 & Glass & 2.3 \\
Gold & 100 & Glass & 2.3 \\
Nickel & 200 & Silicon & 0.5 \\
\hline
\end{tabular}




\section{Periodical pattern formation by laser beam interfer-} ence

Interference of electro-magnetic waves appears when at least two coherent beams propagating at a certain angle to each other interact. In general case the intensity profile in the interference area can be expressed by formula [24]:

$$
I(\overrightarrow{\mathbf{r}}) \propto\left\langle\left(\sum_{i=1}^{N} \overrightarrow{\mathbf{E}}_{i}(\overrightarrow{\mathbf{r}}, t)\right)^{2}\right\rangle,
$$

where $\overrightarrow{\mathbf{r}}$ is the coordinate vector, $i$ is the index of interfering beams, $N$ is the number of the beams, $\overrightarrow{\mathbf{E}}_{i}$ is the electrical field of $i$ beam, $t$ is time, brackets denotes averaging in time at least for one period of radiation. Electrical field of $i$ wave can be expressed as follows:

$$
\overrightarrow{\mathbf{E}}_{i}=\overrightarrow{\mathbf{E}}_{0 i} \cos \left(\overrightarrow{\mathbf{k}}_{i} \cdot \overrightarrow{\mathbf{r}}-\omega t+\varphi_{i}\right),
$$

where $\left|\overrightarrow{\mathbf{E}}_{0 i}\right|$ is the electrical field amplitude of $i$ wave, $\left|\overrightarrow{\mathbf{k}}_{i}\right|=2 \pi / \lambda$ is the wave vector of $i$ wave, $\lambda$ is the wavelength of radiation, $\omega$ is the frequency of radiation, $\varphi_{i}$ is the phase of $i$ wave. If frequency of all the beams is the same the Eqs. (3) and (4) can be simplified as follows [25]:

$$
\begin{aligned}
& I(\mathbf{r}) \propto \frac{1}{2} \sum_{i=1}^{N}\left|\overrightarrow{\mathbf{E}}_{0 i}\right|^{2}+ \\
& \sum_{j<i}^{N} \sum_{i=1}^{N} \overrightarrow{\mathbf{E}}_{0 i} \cdot \overrightarrow{\mathbf{E}}_{0 j} \cos \left(\overrightarrow{\mathbf{k}}_{i} \cdot \overrightarrow{\mathbf{r}}-\overrightarrow{\mathbf{k}}_{j} \cdot \overrightarrow{\mathbf{r}}+\varphi_{i}-\varphi_{j}\right)
\end{aligned}
$$

Periodical intensity field is formed and the period depends on the incident angle between the beams and wavelength. Shape of the pattern is affected by the number of beams and phase difference between them.

\section{Results of patterning in thin metal films by four- beams LBIA}

The periodical intensity distribution of interfering four laser beams calculated by using Eq. (5) is given in Fig. 3.
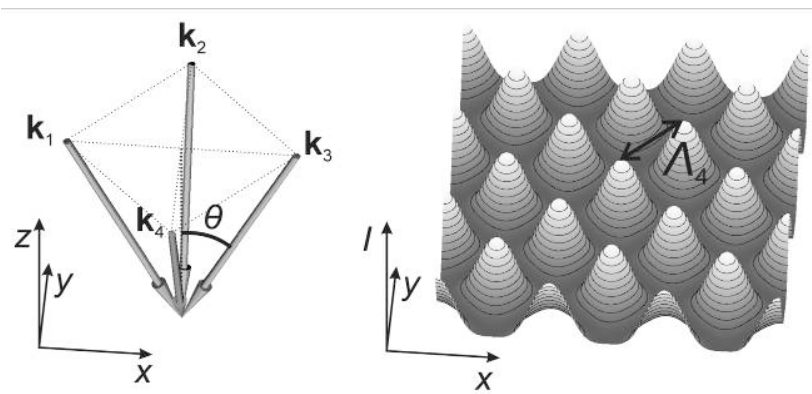

Fig. 3 Wave vectors of four interfering beams (left). Intensity distribution for the interference field of four symmetrically arranged laser beams (right).

The period of the four-beam interference pattern depends on the angle between opposite beams and the wavelength [26]:

$$
\Lambda_{4}=\frac{\lambda}{\sqrt{2} \sin \theta},
$$

where $\theta$ is the half-angle between opposite beams and $\lambda$ is a wavelength of laser radiation.

Laser structuring at high speed over large areas requires that the structure should be ablated with a single laser pulse.
Pulse energy and the number of laser pulses per spot without any shift of a workpiece were used in experiments with thin films of different metals. The front side ablation was used (laser beams were incident from the side of the metal films). Due to 4-beam interference, the resulting structure ablated in the films was quadratic matrix of circular holes with a period predicted by Eq. (1) equal to $\Lambda_{4}=5 \mu \mathrm{m}$. The area where holes were ablated depended on the metal and its thickness. It was as large as $200 \times 200 \mu \mathrm{m}$ in copper and $450 \times 450 \mu \mathrm{m}$ in aluminum films. As the pulse energy available from the used laser was limited to $0.7 \mathrm{~mJ}$, the single pulse ablation of the interference pattern through the whole metal film was achieved in chromium, aluminum and silver The optical microscope images of the structures are presented in Figs. 4-6.

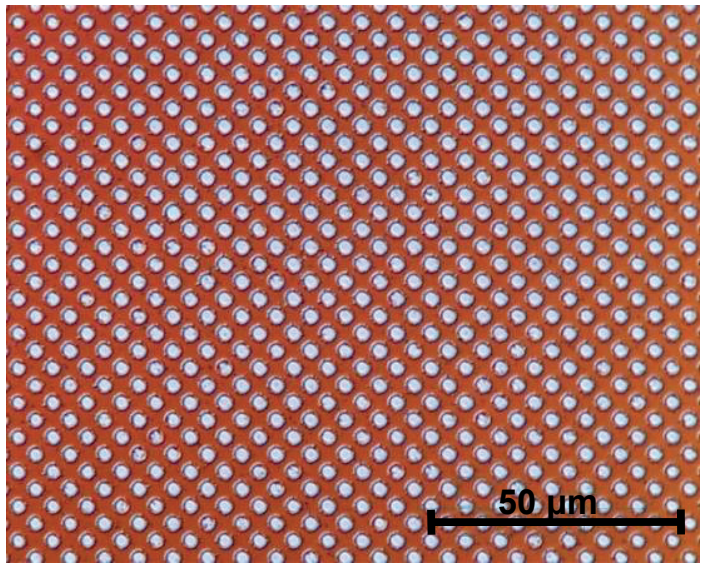

Fig. 4 Matrix of circular holes ablated in chromium film with a single laser pulse using 4-beam interference (laser pulse energy $E_{\mathrm{p}}=0.7 \mathrm{~mJ}$; the structure period $\Lambda_{4}=5 \mu \mathrm{m}$ ).

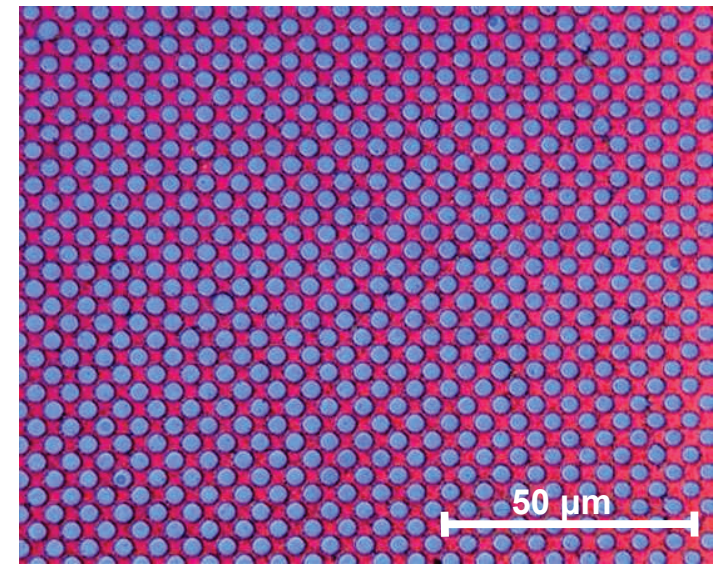

Fig. 5 Matrix of circular holes ablated in aluminum film with a single laser pulse using 4-beam interference (laser pulse energy $E_{\mathrm{p}}=0.7 \mathrm{~mJ}$; the structure period $\Lambda_{4}=5 \mu \mathrm{m}$ ).

The regular structures were formed over the area including a large number of interference maxima and resulting holes were equal in their shape and size. The reason for brown coloring of silver coating in Fig. 6 was the debris of the ablated material. Normally, cleaning in ultrasonic bath removed the debris however silver could not be cleaned in the ultrasonic bath because of the low adhesion to the glass substrate (in ultrasonic bath low adhesion coatings peel off). 


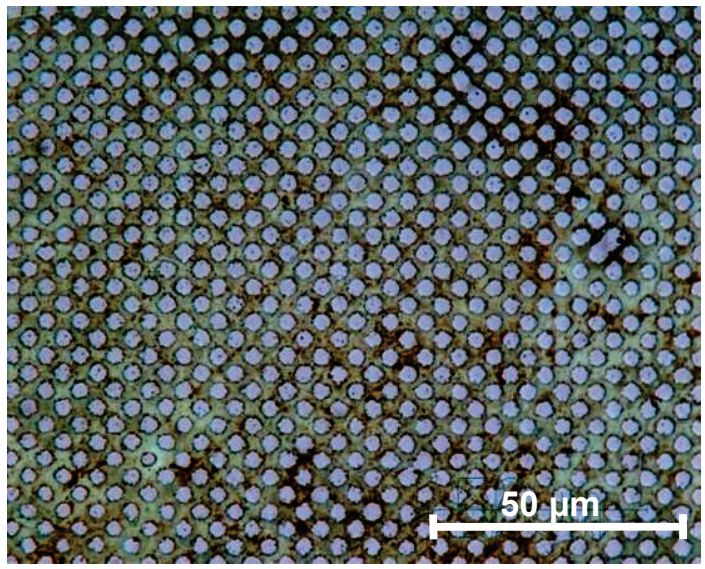

Fig. 6 Matrix of circular holes ablated in silver film with a single laser pulse using 4-beam interference (laser pulse energy $E_{\mathrm{p}}=0.7 \mathrm{~mJ}$; the structure period $\left.\Lambda_{4}=5 \mu \mathrm{m}\right)$.

As gold is a highly reflecting metal, coupling of the laser energy was reduced. Three laser pulses were required to apply per site to ablate the holes at interference maxima through the whole metal film (Fig. 7).

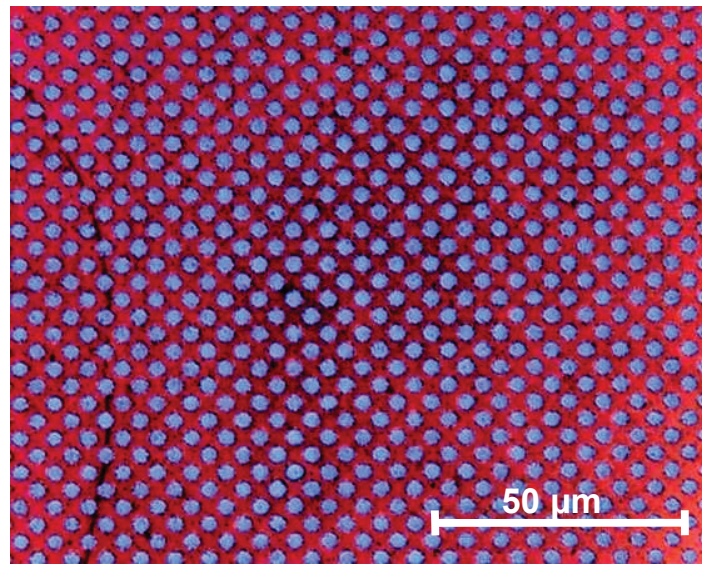

Fig. 7 Matrix of circular holes ablated in gold film with 3 laser pulses using 4-beam interference without any shift (laser pulse energy $E_{\mathrm{p}}=0.7 \mathrm{~mJ}$; the structure period $\Lambda_{4}=5 \mu \mathrm{m}$ ).

Copper film was 5 times thicker and 100 laser pulses were required for the throughout ablation (Fig. 8).

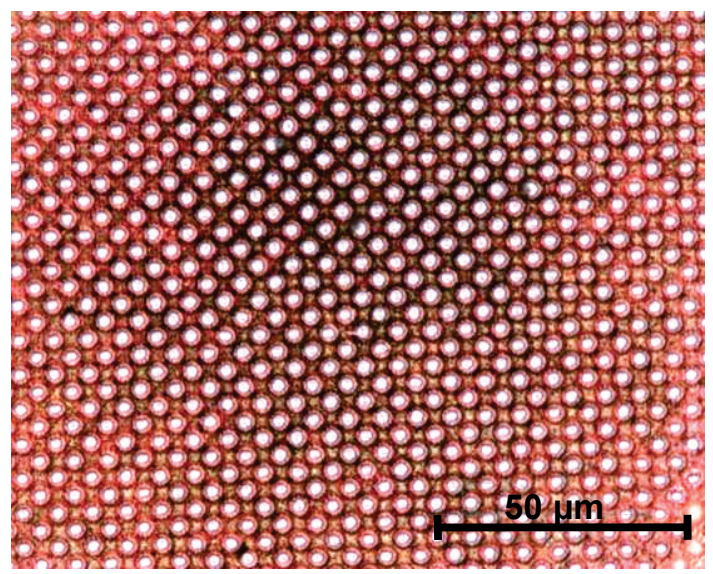

Fig. 8 Matrix of circular holes ablated in copper film with 100 laser pulses using 4-beam interference without any shift (laser pulse energy $E_{\mathrm{p}}=0.7 \mathrm{~mJ}$; the structure period $\left.\Lambda_{4}=5 \mu \mathrm{m}\right)$.
When the phase difference between beam pairs is $\pi / 2$ and the ablation threshold is at $F_{\text {th }} / F_{0}=0.5$ level of intensity at interference maximum, the interference pattern is "chess-board" type. Such structure was fabricated by single pulse ablation in $\mathrm{Cr}$ film by fine adjusting of optics and laser fluence during the experiment (Fig. 9).

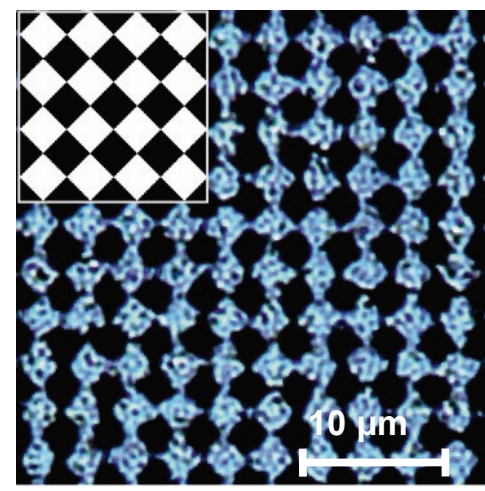

Fig. 9 Picture of "chess-board" type of the structure in Cr film produced with a single-pulse irradiation. Phase difference between beam pairs 1,3 versus 2,4 was $\pi / 2, F_{\mathrm{th}} / F_{0}=0.5$ (insert - modeling).

Periodical structures, consisting of regular arrangement of metal dots with a period, $\Lambda_{4} / \sqrt{2}=3.5 \mu \mathrm{m}$ were fabricated on Cr tin film (Fig. 10).

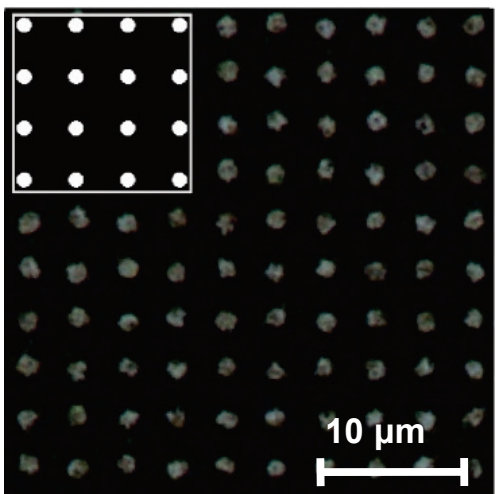

Fig. 10 Dot-like structure in Cr film produced with a single pulse ablation using 4-beam interference. Light areas show remaining metal. Phase difference between beam pairs 1,3 versus 2,4 was $\pi / 2, F_{\mathrm{th}} / F_{0}=0.1$ (insert - modeling).

They can originate from the fine tuning laser intensity to the ablation threshold as well as from transformation of metal segment when it is in a molten state due to surface tension. The case is interesting due to small islands of the remaining metal and reduction of the grating period $\Lambda_{4}$ by square root of two.

When the phase difference between beam pairs is $\pi / 4$ and the ablation threshold is at $F_{\mathrm{th}} / F_{0}=0.1$ level of intensity at interference maximum, the interference pattern consists of elliptical elements arranged in a segmented net with small gaps. Such structure was observed in samples produced with single pulse ablation in Cr film (Fig. 11). 


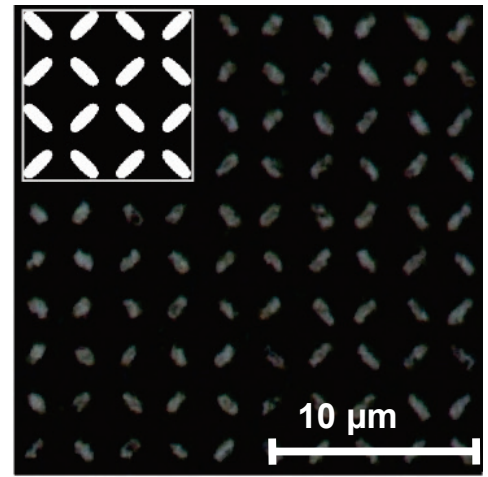

Fig. 11 Net-like structure produced in $\mathrm{Cr}$ film by single pulse ablation. Phase difference between beam pairs 1,3 versus 2,4 was $\pi / 4, F_{\text {th }} / F_{0}=0.1$ (insert - modeling).

When the phase difference between beam pairs is $5 \pi / 18$ and the ablation threshold is at $F_{\mathrm{th}} / F_{0}=0.15$ level of intensity at interference maximum, the interference pattern consists of crosses arranged periodically. Such structure was observed in samples produced by single pulse ablation in Cr film (Fig. 12).

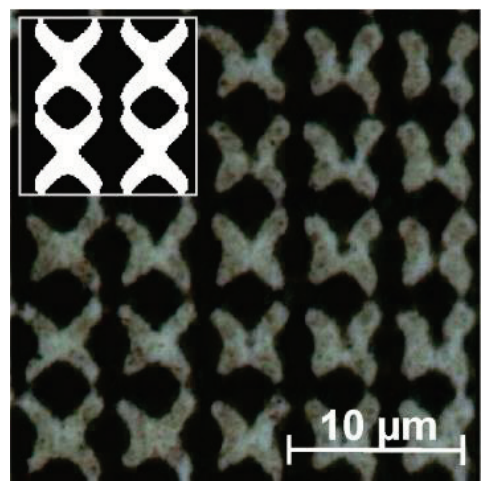

Fig. 12 Cross structure produced in Cr film by single pulse ablation. Phase difference between beam pairs 1,3 versus 2,4 was $5 \pi / 18, F_{\mathrm{th}} / F_{0}=0.15$ (insert - modeling).

5. Results of patterning in thin metal films by sixbeams LBIA

The periodical intensity distribution of interfering six laser beams calculated by using Eq. (5) is given in Fig. 13.
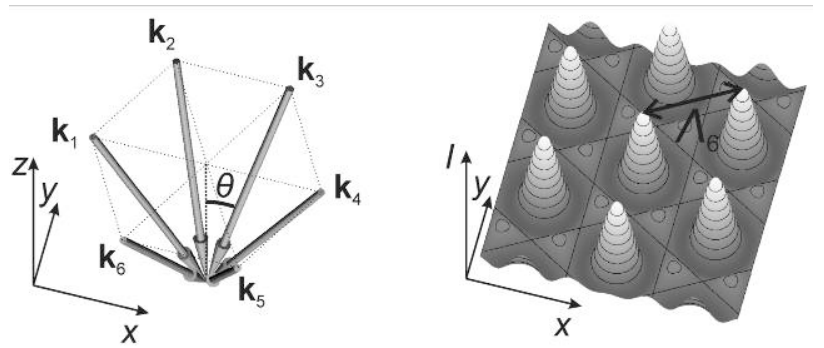

Fig. 13 Wave vectors of six interfering beams (left). Intensity distribution for the interference field of six symmetrically arranged laser beams (right).

The period of the six-beam interference pattern:

$$
\Lambda_{6}=\frac{2 \lambda}{\sqrt{3} \sin \theta},
$$

where $\theta$ is the half-angle between opposite beams.
The hexagonal arrangement of holes with a period predicted by Eq. (2) equal to $\Lambda_{6}=5 \mu \mathrm{m}$ performed on $\mathrm{Cr}$ thin film on glass substrate performed by six-beam LBIA is shown in Fig. 14, Fig. 15 and Fig. 16.

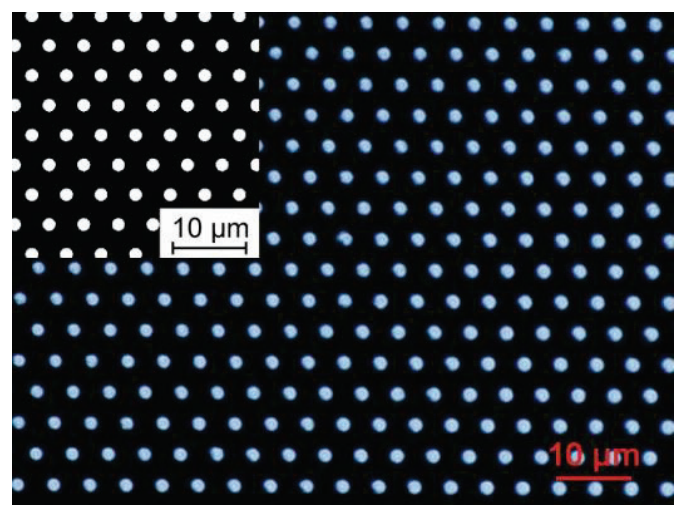

Fig. 14 Hexagonal arrangement of holes ablated in $\mathrm{Cr}$ thin film on glass substrate by using the six-beam LBIA (insert modeling). Number of pulses used was equal to 500, ratio between the peak laser fluence in the intensity profile and the ablation threshold of chromium thin film $F_{\mathrm{th}} / F_{0}=0.38$

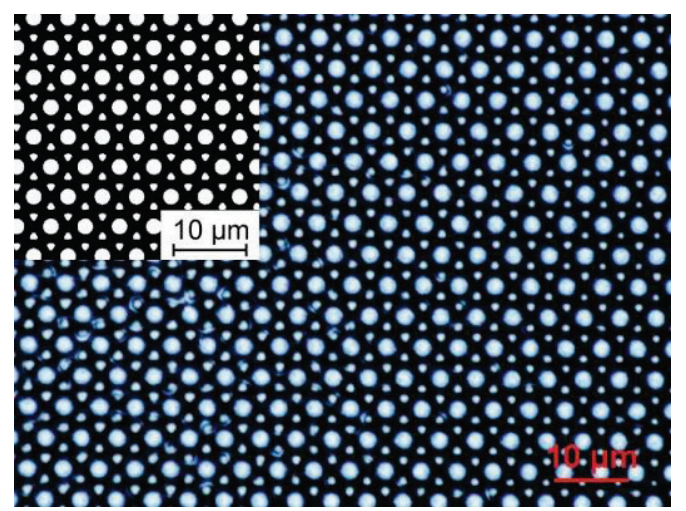

Fig. 15 Hexagonal arrangement of holes each surrounded by hexagonal array of triangles ablated in $\mathrm{Cr}$ thin film on glass substrate by using the six-beam LBIA (insert modeling). Number of pulses used was equal to 500, ratio between the peak laser fluence in the intensity profile and the ablation threshold of chromium thin film $F_{\mathrm{th}} / F_{0}=0.2$.

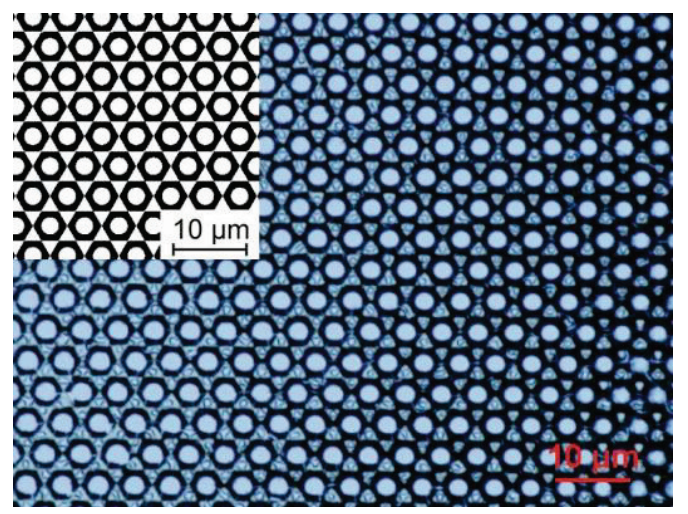

Fig. 16 Ring type structures in hexagonal arrangement ablated in $\mathrm{Cr}$ thin film on glass substrate by using the six-beam LBIA (insert - modeling). Number of pulses used was equal to 400 , ratio between the peak laser fluence in the intensity profile and the ablation threshold of chromium thin film $F_{\mathrm{th}} / F_{0}=0.11$. 


\subsection{Fabrication of C-type structures by the six-beam LBIA}

By varying intensities of separate beams it is possible to generate periodical features which do not have rotational symmetry. The C-type structures were achieved by using the six-beam LBIA in nickel thin film on silicon substrate (Fig. 17).

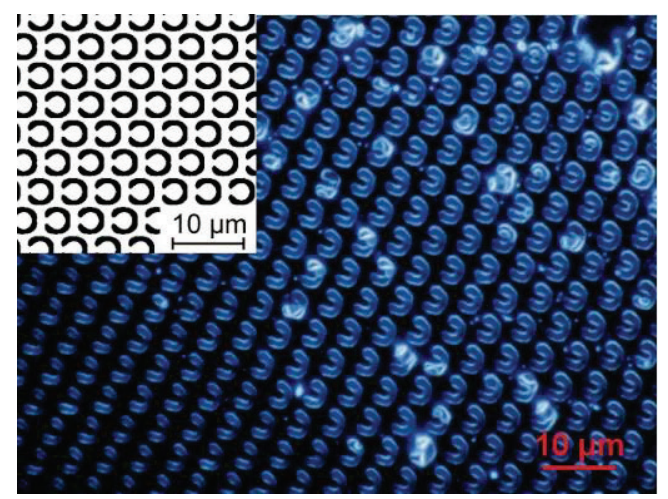

Fig. 17 The $\mathrm{C}-$ type structures ablated in the $\mathrm{Ni}$ thin film on $\mathrm{Si}$ substrate by using the six-beam LBIA (insert - modeling). Number of pulses was equal to 60 . The intensities of the two beams were two times smaller than other four $2 I_{1,2}=I_{3,4,5,6}$. Phase shifts between the beams were equal to $\varphi_{1,2}=\pi / 2, \varphi_{3,4,5,6}=0$.

The shape of the elements, produced by six-beam LBIA is interesting because the split-ring resonators are structural elements for metamaterials with extraordinary response to electro-magnetic waves.

\section{Conclusions}

The interfering laser beam was applied to pattern thin metallic films on the glass substrate and nickel film on the silicon substrate. Periodic structures were fabricated on thin metal films (chromium, aluminum, gold, copper and silver) deposited on the glass substrate using 4-beam laser interference patterning. 2D-gratings with a period of $5 \mu \mathrm{m}$ and consisting of circular holes were fabricated when no phase difference was introduced between symmetrically arranged beams. "Chess-board" and "segmented-net" like periodical structures were fabricated by fine adjustment of the phase difference and laser intensity.

Hexagonal array of holes and ring type structures were achieved by using 6-beams interference ablation on chromium thin film on the glass substrate by controlling the irradiation intensity ratio to the ablation threshold.

The final shape of the structure ablated in metal films was found to be dependent on the phase difference between beam pairs as well as on the irradiation intensity versus the ablation threshold. The material was removed from a substrate in areas where the local intensity (laser fluence) exceeded the threshold value.

The C-type structures were achieved by using the sixbeam interference ablation with intensity control of each laser beam. Such two dimensional periodic structures can be used as polarization-independent high-pass filters in infrared region.

\section{Acknowledgment}

This research was funded by a grant No. ATE-05/2010 from the Research Council of Lithuania.

\section{References}

[1] P. H. Siegel: IEEE Trans. Microwave Theory Tech., 50, (2002) 910 .

[2] B. A. Munk: "Frequency Selective Surfaces: Theory and Design" (Wiley, Toronto, 2000) p.400.

[3] M. Tarasov, V. D. Gromov, G. D. Bogomolov, E. A. Otto and L. S. Kuzmin: Instr. Exper. Techniques, 52, (2009) 74.

[4] J. Koch, F. Korte, C. Fallnich, A. Ostendorf and B. N. Chichkov: Opt. Engineer., 44, (2005) 051103.

[5] J. Reif, F. Costache, M. Henyk and S. V. Pandelov: Appl. Surf. Sci., 197/198, (2002) 891.

[6] Z. Huang, J. E. Carey, M. Liu, X. Guo, E. Mazur and J. C. Campbell: Appl. Phys. Lett., 89, (2006) 033506.

[7] T. Kondo, S. Matsuo, S. Juodkazis and H. Misawa: Appl. Phys. Lett., 79, (2001) 725.

[8] N. Tsutsumi, A. Fujihara and K. Nagata: Thin Solid Films, 517, (2008) 1487.

[9] T. Kondo, S. Juodkazis, V. Mizeikis and H. Misawa: Opt. Express, 14, (2006) 7943.

[10] Q. Xie, M. H. Hong, H. L. Tan, G. X. Chen, L. P. Shi and T. C. Chong: J. Alloys Compounds, 449, (2008) 261.

[11]C. Daniel, F. Mücklich and Z. Liu: Appl. Surf. Sci., 208-209, (2003) 317.

[12] A. F. Lasagni, D. Yuan, P. Shao and S. Das: Adv. Eng. Mat., 11, (2009) 20.

[13]H. C. Guo, D. Nau, A. Radke, X. P. Zhang, J. Stodolka, X. L. Yang, S. G. Tikhodeev, N. A. Gippius and H. Giessen: Appl. Phys. B, 81, (2005) 271.

[14]A. A. Maznev, T. F. Crimmins and K. A. Nelson: Opt. Lett., 23, (1998) 1378.

[15]A. Lasagni and F. Mücklich: Appl. Surf. Sci., 240, (2005) 214.

[16] Y. Nakata, T. Okada and M. Maeda: Appl. Phys. Lett., 81, (2002) 4239.

[17] M. Mäder, T. Höche, J. W. Gerlach, R. Böhme, K. Zimmer and B. Rauschenbach: Phys. Stat. Sol., 2, (2008) 4.

[18]A. Lasagni, C. Holzapfel, T. Weirich and F. Mücklich: Appl. Surf. Sci., 253, (2007) 8070.

[19]X. Li, D. H. Feng, T. Q. Jia, H. Y. He, P. X. Xiong, S. S. Hou, K. Zhou, Z. R. Sun and Z. Z. Xu.: New J. Phys., 12, (2010) 043025.

[20]K. Paivasaari, J. J. Kaakkunen, M. Kuittinen and T. Jaaskelainen: Opt. Express, 15, (2007) 13838.

[21]A. A. Maznev, T. F. Crimmins and K. A. Nelson, How to make femtosecond pulses overlap, Opt. Lett., 23, (1998) 1378.

[22]B. E. A. Saleh and M. C. Teich: "Fundamentals of Photonics", 2nd ed. (Wiley, Boston, 2007) pp.108-156.

[23]E. Molotokaite, M. Gedvilas, G. Račiukaitis and V. Girdauskas: J. Laser Micro/Nanoeng., 5, (2010) 74.

[24] V. Mizeikis, S. Matsuo, S. Juodkazis and H. Misawa: "3D Laser Microfabrication", ed. by H. Misawa and S. Juodkazis, (Wiley-VCH, Weinheim, 2006) p.239.

[25]E. Hecht: "Optics" (Addison Wesley, San Francisco, 2002) p.385.

[26] T. Kondo, S. Matsuo, S. Juodkazis, V. Mizeikis and H. Misawa: Appl. Phys. Lett., 82, (2003) 2758.

(Received: July 23, 2011, Accepted: October 19, 2011) 